\title{
The Clinical Outcomes of Keratoplasty in Irreversible Corneal Decompensation Secondary to Axenfeld-Rieger Syndrome
}

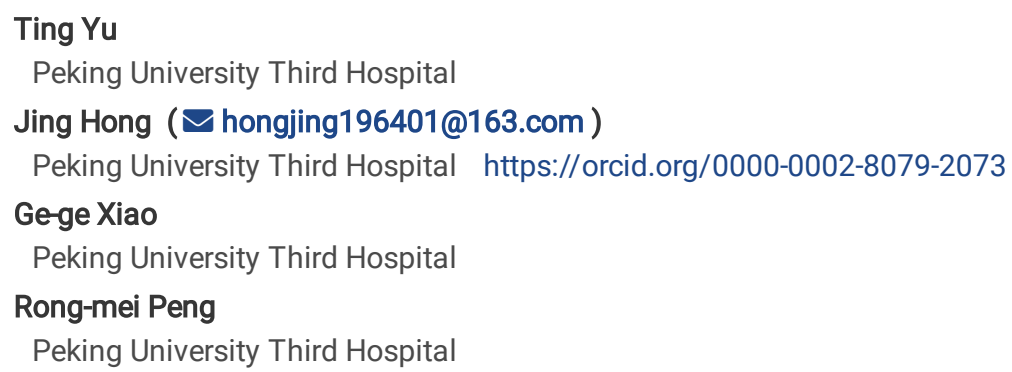

\section{Research Article}

Keywords: Corneal decompensation, Axenfeld-Rieger syndrome, keratoplasty

Posted Date: November 22nd, 2021

DOI: https://doi.org/10.21203/rs.3.rs-1039746/v1

License: (c) (i) This work is licensed under a Creative Commons Attribution 4.0 International License. Read Full License 


\section{Abstract}

Porpose To evaluate the clinical outcomes of penetrating keratoplasty (PK) and Descemet's stripping automated endothelial keratoplasty (DSAEK) in eyes with irreversible corneal decompensation secondary to Axenfeld-Rieger syndrome (ARS).

Methods In this retrospective case series, a total of 4 eyes undergoing PK and 7 eyes undergoing DSAEK, including 1 eye requiring 1 repeat DEASK, between 2014 and 2021 were enrolled. Postoperative complications, graft survival, glaucoma treatment before and after keratoplasty, visual outcomes, and endothelial cell density were recorded.

Results The mean follow-up duration was $33.4 \pm 16.8$ months. Before keratoplasty, the mean BCVA was $2.0 \pm 0.4$ LogMAR, and the mean IOP was $21.6 \pm 8.1 \mathrm{mmHg} .63 .6 \%$ of eyes (7/11) received glaucoma treatment, including 5 eyes with glaucoma surgeries. After keratoplasty, $27.3 \%$ of eyes $(3 / 11)$ exhibited secondary graft failure. The mean BCVA reached a maximum of $0.7 \pm 0.5$ LogMAR at $8.9 \pm 7.5$ months, with no significant difference between the PK and DSAEK groups $\left(P_{1}=1.00, P_{2}=0.12\right)$. Four eyes with previous glaucoma surgeries exhibited markedly high IOP. $72.7 \%$ of eyes (8/11) required additional glaucoma treatments. The mean endothelial cell loss (ECL) rates at $1,6,12$ and 24 months were $43 \%, 49 \%, 63 \%$ and 54\%, respectively, with no significant difference between the $P K$ and DSAEK groups $\left(P_{1}=0.64, P_{2}=1.00, P_{3}=0.57\right.$, and $\left.P_{4}=0.44\right)$.

Conclusion Both PK and DSAEK can successfully treat corneal decompensation secondary to ARS, resulting in similar outcomes with regard to IOP control, BCVA and ECL. IOP control is essential for postoperative management, especially for eyes with previous glaucoma surgeries.

\section{Introduction}

Axenfeld-Rieger syndrome (ARS) is a rare autosomal dominant disease considered part of the ocular anterior segment dysgenesis spectrum, with a prevalence estimated at 1 in 50000 to 100000 newborns [1]. The spectrum of ARS demonstrates locus heterogeneity with two major genes, forkhead box protein C1 (FOXC1, chromosome 6p25) and pituitary homeobox2 (PITX2, chromosome 4q25) [1, 2]. ARS is characterized by varying degrees of anterior segment dysgenesis, with or without craniofacial abnormalities and redundant periumbilical skin [2-5].

Major reported anterior segment features associated with ARS include iris stromal hypoplasia, iridocorneal adhesion, corectopia (distorted or displaced pupils), polycoria (extra holes in the iris), ectropion uveae, and posterior embryotoxon (a prominent, anteriorly displaced Schwalbe's ring near the temporal, posterior corneal limbus with a slit lamp) [4]. Other uncommon systemic anomalies include umbilical hernia, cardiovascular abnormalities and pituitary abnormalities [3].

Patients with ARS have an approximately 50\% risk of developing glaucoma [2]. However, corneal abnormalities are uncommon in ARS [2, 3]. Keratoplasty is taken into account if irreversible corneal decompensation due to corneal endothelial cell loss causes pain and loss of visual acuity, which interfere with a patient's normal activities. For many years, penetrating keratoplasty (PK) has been used for the surgical treatment of corneal endothelial diseases. The most common complications of PK are postoperative astigmatism and unpredictable anisometropia [6]. Descemet's stripping automated endothelial keratoplasty (DSAEK) techniques are increasingly preferred over PK for endothelial dysfunction because of a less invasive process, minimal change in astigmatism, earlier visual rehabilitation, less frequent rejection, lack of suture-associated complications and better tectonic stability while maintaining comparable endothelial cell survival [7-10]. However, DSAEK is more challenging to perform than PK in eyes with ARS because the characteristics restrict the surgeon from working through a small incision to break the iridocorneal adhesion. In addition, iris abnormalities and shallow anterior chambers make graft manipulation and endothelial cell protection more difficult. Keratoplasty was once considered too risky and controversial for patients with ARS, especially pediatric patients, owing to the complicated anterior segment and high risk of secondary glaucoma. However, keratoplasty provides them an opportunity to regain the ability to live a normal life. Several publications have reported the glaucoma management of ARS, but there are very few studies about the outcomes of keratoplasty in patients with ARS $[11,12]$. Whether PK or DSAEK is predominant in the treatment of irreversible corneal decompensation secondary to ARS remains to be elucidated.

The aim of this study was to describe the effect of keratoplasty on graft survival, intraocular pressure (IOP) control, visual acuity, and endothelial cell loss in eyes with irreversible corneal decompensation secondary to ARS and to compare these outcomes between PK and DSAEK.

\section{Methods}

In this retrospective, interventional consecutive case series, we included 11 eyes from 7 patients who were clinically diagnosed with irreversible corneal decompensation secondary to ARS and treated with keratoplasty at the Ophthalmology Department of Peking University Third Hospital from January 2014 to January 2021. The study was conducted in compliance with the principles of the Declaration of Helsinki and approved by the Human Research Ethics Committee of Peking University Third Hospital. Informed consent to use images and data in this study was obtained from each participant or their guardian. 
Patients were diagnosed according to the following inclusion criteria [3-5]: (1) bilateral congenital anterior segment dysgenesis, including iris abnormalities, such as iris stromal hypoplasia, corectopia, polycoria, ectropion uveae, iridocorneal adhesion or posterior embryotoxon; (2) corneal edema and/or opacity; with or without (3) systemic abnormalities, including midface dysmorphism, dental abnormalities, redundant periumbilical skin or other systemic defects.

Preoperative case records were reviewed for the following data: demographics (age at time of keratoplasty, and sex), ocular histories (medical and surgical interventions), best-corrected visual acuity (BCVA) (LogMAR visual chart), IOP (Goldmann tonometer), slit-lamp findings, central corneal thickness (CCT) and anterior chamber depth (ACD) (anterior segment optical coherence tomography) (AS-OCT, Visante, Carl Zeiss Meditec, Dublin, CA, USA), morphological changes and endothelial cell density (ECD) in central endothelial cells (in vivo confocal microscopy) (ICVM, Heideberg Retina Tomograph 3 with Rostock Cornea Module; Heideberg Engineering, GmBH, Dossenheim, Germany), and ECD of the donor tissue (Specular microscope Group I, Class A) (HAI EB-3000 xyz, HAI laboratories, Inc., USA). For LogMAR values worse than 1.6, the following previously described scale was used: counting fingers, 2.0; hand motion, 2.3; light perception, 2.6; and no light perception, 2.9 [13].

\section{Surgical technique}

All keratoplasty operations were performed by a single surgeon $(\mathrm{JH})$. In addition to their own or their guardians' wishes, patients with severe edematous corneas or extremely shallow anterior chambers underwent PK, while the others underwent DSAEK. Referring to the standard procedures, PK (4 grafts, 3 patients) and DSAEK (8 grafts, 5 patients) were performed to replace the full-thickness edematous cornea and the diseased corneal endothelium, respectively $[14,15]$.

Synechiolysis and special considerations for PK. After full-thickness corneal trephination, the iris adhered to the excised cornea was cut with microscissors. Iridocorneal adhesion was carefully broken with viscoelastic (Abbott Medical Optics, Abbott Park, Illinois, USA) or microscissors. Then, the donor tissue was transferred to the host and sutured with 10-0 Prolene sutures. To reduce the risk of peripheral anterior synechiae (PAS) and secondary glaucoma postoperatively, a 0.75 to $1.00 \mathrm{~mm}$ oversized donor cornea was sutured, which depended on each patient's specific requirements.

Synechiolysis and special considerations for DSAEK. An epithelial trephine mark with a diameter of 7.5 or 8.0 mm was made. Viscoelastic from the paracentesis incision was required to break iridocorneal adhesion and maintain the anterior chamber. However, the iris that adhered to the cornea tightly was broken with the mental stab knife. A reverse Terry-Sinskey hook (Bausch and Lomb Surgical, St. Louis, MO, USA) was used to score the endothelium/DM. Then, a Terry Scraper (Bausch and Lomb Surgical) was used to smooth out the rough recipient bed, especially the periphery. Gentle pressure was applied to the inner cornea, taking care not to tear or disrupt corneal stromal fibers. To avoid postoperative PAS and angle obstruction, the $1.5 \mathrm{~mm}$ rim of endothelium/DM within the limbus was left behind. The donor tissue was prepared by a Moria automated lamellar therapeutic keratoplasty microkeratome and associated artificial anterior chamber (Moria Inc. Doylestown, PA, USA). A donor lenticule ranging from 120 to $150 \mu \mathrm{m}$ in thickness was recommended. Removing viscoelastic, the donor lenticule was inserted into the anterior chamber with the suture pull-through insertion technique $[14,15]$ and unfolded in balanced salt solution (BSS, Alcon, Fort Worth, TX, USA). A lenticule-sized bubble was injected from the paracentesis incision, and the donor lenticule was positioned and centered with a roller. The air bubble was maintained for 10 minutes and then reduced to $75 \%$ volume in the anterior chamber. The patient remained supine for 4 hours as required after DSAEK.

\section{Postoperative management}

Topical eye drops, including prednisolone acetate (1.0\%, Allergan, Inc., Irvine, CA, USA), levofloxacin (0.5\%, Santen Co., Ishikawa, Japan) (patients aged 12 years or older) or tobramycin ( $0.3 \%$, Alcon Laboratories, Inc., Fort Worth, TX) (patients younger than 12 years), and artificial tears were administered 4 times daily for the first week, with a gradual decrease as clinically indicated in the following 12 months. Then, a maintenance regimen of low-dose steroids was used from 12 months to 18-24 months after keratoplasty. Tobramycin and dexamethasone eye ointment (tobramycin $0.3 \%$ and dexamethasone $0.1 \%$, Alcon Laboratories, Inc., Fort Worth, TX) was required every night for the first week after PK. Cyclosporin (1\%, North China Pharmaceutical Company, Ltd., Shijiazhuang, Hebei Province, China) (DSAEK) or tacrolimus (0.1\%, Senju Pharmaceutical Co. Ltd., Japan) (PK) was applied 4 times daily from 1 week after keratoplasty, with a gradual decrease as clinically indicated in the following 18 to 24 months. All children were referred to a pediatric ophthalmologist for amblyopia treatment 1 month after keratoplasty.

Patients were routinely evaluated at 1, 3, 7 and 30 days, 3 months, 6 months, and 12 months after keratoplasty and twice per year thereafter. More frequent reviews were performed as necessary. BCVA, IOP, corneal clarity and complications were recorded at each follow-up. ICVM for determining central ECD was performed at 1, 6, and 12 months and once per year thereafter. Graft rejection, primary graft failure and secondary graft failure were described in detail previously $[16,17]$. The values were excluded from the following analysis in the presence of irreversible corneal decompensation and repeat keratoplasty.

\section{Statistical analysis}


The endothelial cell loss (ECL) rate was calculated by subtracting postoperative ECD from baseline donor ECD and then dividing by baseline donor ECD and multiplying by 100 . The distribution of the data was assessed for normality via the Kolmogorov-Smirnoff-Lillefors test. Data are presented as the mean \pm standard deviation. The data with a normal distribution were estimated using a t-test. Analysis for data with nonnormal distributions was performed using the Wilcoxon Mann-Whitney or Wilcoxon signed rank test as appropriate. Categorical variables were compared using Chi-square/Fisher's exact test. The potential association between the 6-month ECL rate and the 12-month ECL rate and previous glaucoma surgeries, baseline IOP, ACD before keratoplasty, type of keratoplasty, and additional glaucoma surgeries after keratoplasty was assessed by analysis of variance. A two-sided P value $<0.05$ was considered statistically significant. Statistical analysis was performed using IBM SPSS statistics, version 24.0 (SPSS, Inc., Chicago, IL, USA).

\section{Results}

Eleven eyes of 7 patients were included in the study. Family history was positive for ARS in 1 patient (Patient 6). Two patients underwent genetic counseling, and genomic DNA from the patients' blood samples was PCR-amplified and screened for mutations in PITX2 c.253 A>G (Patient 3 ) and PITX2 c. 286 C>T(Q96X) (Patient 6). Physical examinations revealed craniofacial abnormalities in all patients and redundant periumbilical skin in 4 patients. The mean age was $17.7 \pm 12.6$ years (range, 6.3-39.8 years) at the time of first keratoplasty, and 5 ( $71.4 \%$ ) were male. The mean follow-up duration was $33.4 \pm 16.8$ months. There was no significant difference between the PK group ( $n=4,36.4 \%$ ) and DSAEK group ( $n=7$, $63.6 \%)(44.5 \pm 14.8$ months vs. $28.6 \pm 16.0$ months, $P=0.13)$.

Before keratoplasty, the mean BCVA was 2.0 \pm 0.4 LogMAR (range, 1.1-2.6 LogMAR), and the mean IOP was 21.6 $\pm 8.1 \mathrm{mmHg}$ (range, $12-36$ $\mathrm{mmHg})$. There was no significant difference between the PK and DSAEK groups in the BCVA (2.1 \pm 0.2 LogMAR vs. 2.0 $\pm 0.5 \mathrm{LogMAR}, \mathrm{P}=1.00)$ and IOP $(17.0 \pm 6.3 \mathrm{mmHg}$ vs. $24.3 \pm 8.2 \mathrm{mmHg}, \mathrm{P}=0.13)$. One PK eye and 4 DSAEK eyes showed elevated IOP (> $21 \mathrm{mmHg})$. One of $4 \mathrm{PK}$ eyes (25.0\%) required topical glaucoma medications and surgeries; however, 6 of 7 DSAEK eyes (85.7\%) received glaucoma treatment: 2 of these (33.3\%) were on topical medications alone, while 4 (66.7\%) had previous glaucoma surgeries. One eye with lens anterior dislocation underwent combined cataract extraction-synechiolysis-gonioplasty-anterior vitrectomy-posterior chamber intraocular lens (PCIOL) implantation, followed by an elevated IOP (Patient 3, right eye) (Table 1). 
Table 1

Clinical information before and after keratoplasty

\begin{tabular}{|c|c|c|c|c|c|c|c|c|c|c|c|}
\hline \multirow[t]{2}{*}{ No. } & \multirow{2}{*}{$\begin{array}{l}\text { Age/ } \\
\text { sex }\end{array}$} & \multirow[t]{2}{*}{ Eye } & \multirow{2}{*}{$\begin{array}{l}\text { Ocular } \\
\text { abnormalities }\end{array}$} & \multirow[t]{2}{*}{ Procedure } & \multicolumn{2}{|c|}{ BCVA (LogMAR) } & \multicolumn{2}{|c|}{ IOP control } & \multirow{2}{*}{$\begin{array}{l}\text { Postop } \\
\text { CDR } \\
\text { (after } \\
\text { cleared } \\
\text { graft) }\end{array}$} & \multirow{2}{*}{$\begin{array}{l}\text { Time } \\
\text { to } \\
\text { failure } \\
\text { (mo) }\end{array}$} & \multirow{2}{*}{$\begin{array}{l}\mathrm{FU} \\
\text { (mo) }\end{array}$} \\
\hline & & & & & Baseline & $\begin{array}{l}\text { Postop } \\
\text { maximum }\end{array}$ & $\begin{array}{l}\text { Baseline } \\
\text { (No. of } \\
\text { GM } \\
+ \text { GS) }\end{array}$ & $\begin{array}{l}\text { Postop IOP } \\
\text { problems; } \\
\text { additional GM + } \\
\text { GS }\end{array}$ & & & \\
\hline \multirow[t]{14}{*}{1} & \multirow[t]{7}{*}{$6 / F$} & \multirow[t]{7}{*}{ OD } & $\begin{array}{l}\text { Cornea: } \\
\text { edema + } \\
\text { opaque } \\
\text { (central) }\end{array}$ & \multirow[t]{7}{*}{ PK-SL } & \multirow[t]{7}{*}{2.0} & \multirow[t]{7}{*}{0.3} & \multirow[t]{7}{*}{12 (Nil) } & \multirow[t]{7}{*}{ No } & \multirow[t]{7}{*}{0.3} & \multirow[t]{7}{*}{ - } & \multirow[t]{7}{*}{61} \\
\hline & & & $\begin{array}{l}\text { Posterior } \\
\text { embryotoxon: } \\
(-)\end{array}$ & & & & & & & & \\
\hline & & & ССТ: $1.22 \mathrm{~mm}$ & & & & & & & & \\
\hline & & & $\begin{array}{l}\text { Iris: } \\
\text { corectopia, IH, } \\
\text { AS }\end{array}$ & & & & & & & & \\
\hline & & & ACD: $0.44 \mathrm{~mm}$ & & & & & & & & \\
\hline & & & $\begin{array}{l}\text { Comorbidities: } \\
(-)\end{array}$ & & & & & & & & \\
\hline & & & MEC: ND & & & & & & & & \\
\hline & & \multirow[t]{7}{*}{ OS } & $\begin{array}{l}\text { Cornea: } \\
\text { edema + } \\
\text { opaque } \\
\text { (central) }\end{array}$ & \multirow[t]{7}{*}{ PK-SL } & \multirow[t]{7}{*}{2.0} & \multirow[t]{7}{*}{0.2} & \multirow[t]{7}{*}{12 (Nil) } & \multirow[t]{7}{*}{$\begin{array}{l}\text { Yes; raised at } \\
16 \text { mo, } \\
\text { controlled on } 2 \\
\text { GM }\end{array}$} & \multirow[t]{7}{*}{0.3} & \multirow[t]{7}{*}{-} & \multirow[t]{7}{*}{53} \\
\hline & & & $\begin{array}{l}\text { Posterior } \\
\text { embryotoxon: } \\
(-)\end{array}$ & & & & & & & & \\
\hline & & & ССТ: $0.82 \mathrm{~mm}$ & & & & & & & & \\
\hline & & & $\begin{array}{l}\text { Iris: } \\
\text { corectopia, IH, } \\
\text { AS }\end{array}$ & & & & & & & & \\
\hline & & & ACD: $0.55 \mathrm{~mm}$ & & & & & & & & \\
\hline & & & $\begin{array}{l}\text { Comorbidities: } \\
(-)\end{array}$ & & & & & & & & \\
\hline & & & MEC: ND & & & & & & & & \\
\hline \multirow[t]{7}{*}{2} & \multirow[t]{7}{*}{$7 / \mathrm{M}$} & & $\begin{array}{l}\text { Cornea: } \\
\text { edema + ulcer } \\
\text { + opaque } \\
\text { (diffuse) }\end{array}$ & PK-SL & 2.3 & 1.0 & 19 (Nil) & No & 0.3 & - & 31 \\
\hline & & & $\begin{array}{l}\text { Posterior } \\
\text { embryotoxon: } \\
\text { ND }\end{array}$ & & & & & & & & \\
\hline & & & ССТ: $1.44 \mathrm{~mm}$ & & & & & & & & \\
\hline & & & $\begin{array}{l}\text { Iris: } \\
\text { corectopia, } \\
\text { polycoria, IH, } \\
\text { AS }\end{array}$ & & & & & & & & \\
\hline & & & ACD: $1.48 \mathrm{~mm}$ & & & & & & & & \\
\hline & & & $\begin{array}{l}\text { Comorbidities: } \\
\text { microcornea, } \\
\text { high myopia }\end{array}$ & & & & & & & & \\
\hline & & & MEC: ND & & & & & & & & \\
\hline
\end{tabular}




\begin{tabular}{|c|c|c|c|c|c|c|c|c|c|c|c|}
\hline \multirow[t]{2}{*}{ No. } & \multirow{2}{*}{$\begin{array}{l}\text { Age/ } \\
\text { sex }\end{array}$} & \multirow[t]{2}{*}{ Eye } & \multirow{2}{*}{$\begin{array}{l}\text { Ocular } \\
\text { abnormalities }\end{array}$} & \multirow[t]{2}{*}{ Procedure } & \multicolumn{2}{|c|}{ BCVA (LogMAR) } & \multicolumn{2}{|c|}{ IOP control } & \multirow{2}{*}{$\begin{array}{l}\text { Postop } \\
\text { CDR } \\
\text { (after } \\
\text { cleared } \\
\text { graft) }\end{array}$} & \multirow{2}{*}{$\begin{array}{l}\text { Time } \\
\text { to } \\
\text { failure } \\
\text { (mo) }\end{array}$} & \multirow{2}{*}{$\begin{array}{l}\mathrm{FU} \\
\text { (mo) }\end{array}$} \\
\hline & & & & & Baseline & $\begin{array}{l}\text { Postop } \\
\text { maximum }\end{array}$ & $\begin{array}{l}\text { Baseline } \\
\text { (No. of } \\
\text { GM } \\
+ \text { GS) }\end{array}$ & $\begin{array}{l}\text { Postop IOP } \\
\text { problems; } \\
\text { additional GM + } \\
\text { GS }\end{array}$ & & & \\
\hline \multirow[t]{14}{*}{3} & \multirow[t]{7}{*}{$11 / \mathrm{M}$} & \multirow[t]{7}{*}{$O D$} & $\begin{array}{l}\text { Cornea: } \\
\text { edema + } \\
\text { opaque } \\
\text { (diffuse) }\end{array}$ & \multirow[t]{7}{*}{ PK-SL } & \multirow[t]{7}{*}{2.0} & \multirow[t]{7}{*}{1.1} & \multirow[t]{7}{*}{$\begin{array}{l}25(2 ; \\
\text { CPC } \times 2+ \\
\text { GDD })\end{array}$} & \multirow{7}{*}{$\begin{array}{l}\text { Yes; raised at } 1 \\
\text { mo, controlled } \\
\text { on CPC + GDD } \\
+ \text { combined } \\
\text { CLASS- } \\
\text { trabeculectomy- } \\
\text { trabeculotomy } \\
\text { at } 14 \text { mo }\end{array}$} & \multirow[t]{7}{*}{0.8} & \multirow[t]{7}{*}{21} & \multirow[t]{7}{*}{33} \\
\hline & & & $\begin{array}{l}\text { Posterior } \\
\text { embryotoxon: } \\
(+)\end{array}$ & & & & & & & & \\
\hline & & & ССТ: 1.32 mm & & & & & & & & \\
\hline & & & $\begin{array}{l}\text { Iris: } \\
\text { corectopia, } \\
\text { polycoria, IH, } \\
\text { AS }\end{array}$ & & & & & & & & \\
\hline & & & ACD: $2.92 \mathrm{~mm}$ & & & & & & & & \\
\hline & & & $\begin{array}{l}\text { Comorbidities: } \\
\text { glaucoma }\end{array}$ & & & & & & & & \\
\hline & & & MEC: ND & & & & & & & & \\
\hline & & \multirow[t]{7}{*}{ os } & $\begin{array}{l}\text { Cornea: } \\
\text { edema + } \\
\text { opaque } \\
\text { (peripheral) }\end{array}$ & \multirow[t]{7}{*}{$\begin{array}{l}\text { DSAEK- } \\
\text { SL }\end{array}$} & \multirow[t]{7}{*}{1.1} & \multirow[t]{7}{*}{0.3} & \multirow[t]{7}{*}{$24(2)$} & \multirow[t]{7}{*}{ No } & \multirow[t]{7}{*}{0.4} & \multirow[t]{7}{*}{-} & \multirow[t]{7}{*}{29} \\
\hline & & & $\begin{array}{l}\text { Posterior } \\
\text { embryotoxon: } \\
(+)\end{array}$ & & & & & & & & \\
\hline & & & ССТ: $1.03 \mathrm{~mm}$ & & & & & & & & \\
\hline & & & $\begin{array}{l}\text { Iris: } \\
\text { corectopia, IH, } \\
\text { AS }\end{array}$ & & & & & & & & \\
\hline & & & ACD: $1.88 \mathrm{~mm}$ & & & & & & & & \\
\hline & & & $\begin{array}{l}\text { Comorbidities: } \\
\text { glaucoma }\end{array}$ & & & & & & & & \\
\hline & & & $\begin{array}{l}\text { MEC: ND } \\
\text { (regular } \\
\text { hexagonal } \\
\text { ECs with } \\
\text { bright nuclei } \\
\text { were detected } \\
\text { before corneal } \\
\text { edema) }\end{array}$ & & & & & & & & \\
\hline \multirow[t]{6}{*}{4} & \multirow[t]{6}{*}{$7 / M$} & $\mathrm{OD}$ & $\begin{array}{l}\text { Cornea: } \\
\text { edema } \\
\text { +opaque } \\
\text { (peripheral) }\end{array}$ & $\begin{array}{l}\text { DSAEK- } \\
\text { SL }\end{array}$ & 1.6 & 0.5 & $13(1)$ & $\begin{array}{l}\text { Yes, raised at } 1 \\
\text { mo, controlled } \\
\text { on } 2 \mathrm{GM}\end{array}$ & 0.4 & - & 41 \\
\hline & & & $\begin{array}{l}\text { Posterior } \\
\text { embryotoxon: } \\
(+)\end{array}$ & & & & & & & & \\
\hline & & & ССТ: $0.88 \mathrm{~mm}$ & & & & & & & & \\
\hline & & & $\begin{array}{l}\text { Iris: } \\
\text { corectopia, } \\
\text { polycoria, IH, } \\
\text { AS }\end{array}$ & & & & & & & & \\
\hline & & & ACD: $1.07 \mathrm{~mm}$ & & & & & & & & \\
\hline & & & $\begin{array}{l}\text { Comorbidities: } \\
(-)\end{array}$ & & & & & & & & \\
\hline
\end{tabular}




\begin{tabular}{|c|c|c|c|c|c|c|c|c|c|c|c|}
\hline \multirow[t]{2}{*}{ No. } & \multirow{2}{*}{$\begin{array}{l}\text { Age/ } \\
\text { sex }\end{array}$} & \multirow[t]{2}{*}{ Eye } & \multirow{2}{*}{$\begin{array}{l}\text { Ocular } \\
\text { abnormalities }\end{array}$} & \multirow[t]{2}{*}{ Procedure } & \multicolumn{2}{|c|}{ BCVA (LogMAR) } & \multicolumn{2}{|c|}{ IOP control } & \multirow{2}{*}{$\begin{array}{l}\text { Postop } \\
\text { CDR } \\
\text { (after } \\
\text { cleared } \\
\text { graft) }\end{array}$} & \multirow{2}{*}{$\begin{array}{l}\text { Time } \\
\text { to } \\
\text { failure } \\
\text { (mo) }\end{array}$} & \multirow{2}{*}{$\begin{array}{l}\text { FU } \\
\text { (mo) }\end{array}$} \\
\hline & & & & & Baseline & $\begin{array}{l}\text { Postop } \\
\text { maximum }\end{array}$ & $\begin{array}{l}\text { Baseline } \\
\text { (No. of } \\
\text { GM } \\
+ \text { GS) }\end{array}$ & $\begin{array}{l}\text { Postop IOP } \\
\text { problems; } \\
\text { additional GM + } \\
\text { GS }\end{array}$ & & & \\
\hline & & & $\begin{array}{l}\text { MEC: irregular } \\
\text { hexagonal } \\
\text { ECs }\end{array}$ & & & & & & & & \\
\hline & & OS & $\begin{array}{l}\text { Cornea: } \\
\text { edema + } \\
\text { opaque } \\
\text { (peripheral) }\end{array}$ & $\begin{array}{l}\text { 1. DSAEK- } \\
\text { SL }\end{array}$ & 2.0 & 0.2 & 20 (Nil) & $\begin{array}{l}\text { Yes, raised at } 1 \\
\text { mo, controlled } \\
\text { on } 2 \mathrm{GM}\end{array}$ & 0.4 & 24 & 38 \\
\hline & & & $\begin{array}{l}\text { Posterior } \\
\text { embryotoxon: } \\
(-)\end{array}$ & & & & & & & & \\
\hline & & & ССТ: $0.94 \mathrm{~mm}$ & & & & & & & & \\
\hline & & & $\begin{array}{l}\text { Iris: } \\
\text { corectopia, IH, } \\
\text { AS }\end{array}$ & $\begin{array}{l}\text { 2. DSAEK- } \\
\text { SL }\end{array}$ & 1.1 & 0.4 & $16(2)$ & No & 0.4 & - & 9 \\
\hline & & & ACD: $1.33 \mathrm{~mm}$ & & & & & & & & \\
\hline & & & $\begin{array}{l}\text { Comorbidities: } \\
(-)\end{array}$ & & & & & & & 47 total & \\
\hline & & & $\begin{array}{l}\text { MEC: irregular } \\
\text { hexagonal } \\
\text { ECs }\end{array}$ & & & & & & & & \\
\hline \multirow[t]{7}{*}{5} & \multirow[t]{7}{*}{$21 / \mathrm{M}$} & \multirow[t]{7}{*}{ OS } & $\begin{array}{l}\text { Cornea: } \\
\text { edema + } \\
\text { opaque } \\
\text { (peripheral) + } \\
\text { pannus }\end{array}$ & \multirow[t]{7}{*}{$\begin{array}{l}\text { DSAEK- } \\
\text { SL- } \\
\text { Phaco- } \\
\text { PCIOL }\end{array}$} & \multirow[t]{7}{*}{2.6} & \multirow[t]{7}{*}{2.0} & \multirow[t]{7}{*}{$\begin{array}{l}17 \\
\text { (trabecul- } \\
\text { ectomy) }\end{array}$} & \multirow[t]{7}{*}{$\begin{array}{l}\text { Yes; raised at } 2 \\
\text { mo, poorly } \\
\text { controlled with } \\
3 \mathrm{GM}+\mathrm{PCIOL} \\
\text { explantation-AV } \\
+\mathrm{CPC}\end{array}$} & \multirow[t]{7}{*}{0.8} & \multirow[t]{7}{*}{-} & \multirow[t]{7}{*}{15} \\
\hline & & & $\begin{array}{l}\text { Posterior } \\
\text { embryotoxon: } \\
(+)\end{array}$ & & & & & & & & \\
\hline & & & ССТ: $0.73 \mathrm{~mm}$ & & & & & & & & \\
\hline & & & $\begin{array}{l}\text { Iris: } \\
\text { corectopia, } \\
\text { polycoria, IH, } \\
\text { AS, EU }\end{array}$ & & & & & & & & \\
\hline & & & ACD: $1.56 \mathrm{~mm}$ & & & & & & & & \\
\hline & & & $\begin{array}{l}\text { Comorbidities: } \\
\text { glaucoma, } \\
\text { cataract }\end{array}$ & & & & & & & & \\
\hline & & & MEC: ND & & & & & & & & \\
\hline \multirow[t]{5}{*}{6} & \multirow[t]{5}{*}{$40 / F$} & \multirow[t]{5}{*}{$\mathrm{OD}$} & $\begin{array}{l}\text { Cornea: } \\
\text { edema + } \\
\text { opaque } \\
\text { (peripheral) + } \\
\text { pannus }\end{array}$ & \multirow[t]{5}{*}{$\begin{array}{l}\text { DSAEK- } \\
\text { SL- } \\
\text { Phaco- } \\
\text { PCIOL }\end{array}$} & \multirow[t]{5}{*}{2.0} & \multirow[t]{5}{*}{0.4} & \multirow[t]{5}{*}{$\begin{array}{l}30(1 ; \\
\text { CPC×2) }\end{array}$} & \multirow[t]{5}{*}{$\begin{array}{l}\text { Yes; controlled } \\
\text { on } 3 \mathrm{GM}+\mathrm{CPC} \\
+\mathrm{GDD} \text { at } 33 \mathrm{mo}\end{array}$} & \multirow[t]{5}{*}{0.9} & \multirow[t]{5}{*}{45} & \multirow[t]{5}{*}{45} \\
\hline & & & $\begin{array}{l}\text { Posterior } \\
\text { embryotoxon: } \\
(+)\end{array}$ & & & & & & & & \\
\hline & & & ССТ: $0.77 \mathrm{~mm}$ & & & & & & & & \\
\hline & & & $\begin{array}{l}\text { Iris: } \\
\text { corectopia, } \\
\text { polycoria, IH, } \\
\text { AS, EU }\end{array}$ & & & & & & & & \\
\hline & & & ACD: $1.84 \mathrm{~mm}$ & & & & & & & & \\
\hline
\end{tabular}




\begin{tabular}{|c|c|c|c|c|c|c|c|c|c|c|c|}
\hline \multirow[t]{2}{*}{ No. } & \multirow{2}{*}{$\begin{array}{l}\text { Age/ } \\
\text { sex }\end{array}$} & \multirow[t]{2}{*}{ Eye } & \multirow{2}{*}{$\begin{array}{l}\text { Ocular } \\
\text { abnormalities }\end{array}$} & \multirow[t]{2}{*}{ Procedure } & \multicolumn{2}{|c|}{ BCVA (LogMAR) } & \multicolumn{2}{|l|}{ IOP control } & \multirow{2}{*}{$\begin{array}{l}\text { Postop } \\
\text { CDR } \\
\text { (after } \\
\text { cleared } \\
\text { graft) }\end{array}$} & \multirow{2}{*}{$\begin{array}{l}\text { Time } \\
\text { to } \\
\text { failure } \\
\text { (mo) }\end{array}$} & \multirow{2}{*}{$\begin{array}{l}\text { FU } \\
\text { (mo) }\end{array}$} \\
\hline & & & & & Baseline & $\begin{array}{l}\text { Postop } \\
\text { maximum }\end{array}$ & $\begin{array}{l}\text { Baseline } \\
\text { (No. of } \\
\text { GM } \\
+ \text { GS) }\end{array}$ & $\begin{array}{l}\text { Postop IOP } \\
\text { problems; } \\
\text { additional GM + } \\
\text { GS }\end{array}$ & & & \\
\hline & & & $\begin{array}{l}\text { Comorbidities: } \\
\text { glaucoma, } \\
\text { cataract, high } \\
\text { myopia }\end{array}$ & & & & & & & & \\
\hline & & & MEC: ND & & & & & & & & \\
\hline \multirow[t]{14}{*}{7} & \multirow[t]{7}{*}{$32 / \mathrm{M}$} & \multirow[t]{7}{*}{ OD } & $\begin{array}{l}\text { Cornea: } \\
\text { edema + } \\
\text { opaque } \\
\text { (peripheral) }\end{array}$ & \multirow[t]{7}{*}{$\begin{array}{l}\text { DSAEK- } \\
\text { SL }\end{array}$} & \multirow[t]{7}{*}{2.3} & \multirow[t]{7}{*}{0.5} & \multirow[t]{7}{*}{30 (GDD) } & \multirow[t]{7}{*}{$\begin{array}{l}\text { Yes, controlled } \\
\text { on } 2 \mathrm{GM}\end{array}$} & \multirow[t]{7}{*}{0.6} & \multirow[t]{7}{*}{-} & \multirow[t]{7}{*}{11} \\
\hline & & & $\begin{array}{l}\text { Posterior } \\
\text { embryotoxon: } \\
(+)\end{array}$ & & & & & & & & \\
\hline & & & ССТ: $0.95 \mathrm{~mm}$ & & & & & & & & \\
\hline & & & $\begin{array}{l}\text { Iris: } \\
\text { corectopia, IH, } \\
\text { AS }\end{array}$ & & & & & & & & \\
\hline & & & ACD: 2.59 mm & & & & & & & & \\
\hline & & & $\begin{array}{l}\text { Comorbidities: } \\
\text { glaucoma }\end{array}$ & & & & & & & & \\
\hline & & & $\begin{array}{l}\text { MEC: ND } \\
\text { (regular } \\
\text { hexagonal } \\
\text { ECs with } \\
\text { bright nuclei } \\
\text { were detected } \\
\text { before corneal } \\
\text { edema) }\end{array}$ & & & & & & & & \\
\hline & & \multirow[t]{7}{*}{ OS } & $\begin{array}{l}\text { Cornea: } \\
\text { edema + } \\
\text { opaque } \\
\text { (central) }\end{array}$ & \multirow[t]{7}{*}{$\begin{array}{l}\text { DSAEK- } \\
\text { SL }\end{array}$} & \multirow[t]{7}{*}{2.3} & \multirow[t]{7}{*}{0.8} & \multirow[t]{7}{*}{$\begin{array}{l}36(3 ; \\
\text { GDD + } \\
\text { tube } \\
\text { explantat- } \\
\text { ion) }\end{array}$} & \multirow[t]{7}{*}{$\begin{array}{l}\text { Yes, poorly } \\
\text { controlled with } \\
3 \mathrm{GM}\end{array}$} & \multirow[t]{7}{*}{0.8} & \multirow[t]{7}{*}{-} & \multirow[t]{7}{*}{12} \\
\hline & & & $\begin{array}{l}\text { Posterior } \\
\text { embryotoxon: } \\
(-)\end{array}$ & & & & & & & & \\
\hline & & & ССТ: 1.09 mm & & & & & & & & \\
\hline & & & $\begin{array}{l}\text { Iris: } \\
\text { corectopia, IH, } \\
\text { AS }\end{array}$ & & & & & & & & \\
\hline & & & ACD: 1.77 mm & & & & & & & & \\
\hline & & & $\begin{array}{l}\text { Comorbidities: } \\
\text { glaucoma }\end{array}$ & & & & & & & & \\
\hline & & & MEC: ND & & & & & & & & \\
\hline $\begin{array}{l}\text { No.= } \\
\text { GS }= \\
\text { thick } \\
\text { of th } \\
\text { auto } \\
\text { CPC } \\
\text { filter }\end{array}$ & $\begin{array}{l}\text { tient n } \\
\text { aucoms } \\
\text { ess; AC } \\
\text { ris; EU: } \\
\text { ated en } \\
\text { ycloph } \\
\text { g bleb; }\end{array}$ & $\begin{array}{l}\text { iber; } \\
\text { urge } \\
\text { ante } \\
\text { trop } \\
\text { theli } \\
\text { coa } \\
\text { =ant }\end{array}$ & $\begin{array}{l}\text { CVA=best-correc } \\
\text { es; } C D R=\text { cup dis } \\
\text { ior chamber depth } \\
\text { on uveae; ND=not } \\
\text { l keratoplasty; SL } \\
\text { ulation; GDD=gla } \\
\text { rior vitrectomy. C }\end{array}$ & $\begin{array}{l}\text { d visual acu } \\
\text { atio; mo= m } \\
\text { MEC=morpł } \\
\text { etectable; E } \\
\text { synechiolys } \\
\text { oma draina } \\
\text { and ACD n }\end{array}$ & $\begin{array}{l}\text { ty; postop= } \\
\text { onths; FU=f } \\
\text { ological ch } \\
=\text { endothelia } \\
\text {; Phaco=p } \\
\text { je devices; } \\
\text { ere measure }\end{array}$ & $\begin{array}{l}\text { stoperative } \\
\text { low-up; } M=1 \\
\text { iges in endo } \\
\text { cells; } P K=p \\
\text { coemulsific } \\
\text { ASS }=\mathrm{CO}_{2} \\
\text { with } \mathrm{AS}-\mathrm{O}\end{array}$ & $\begin{array}{l}=\text { intraocular } \\
\mathrm{F}=\text { female; } \\
\text { al cells; } I \mathrm{H}=\mathrm{i} \\
\text { ating kerato } \\
\text {; } P C I O L=p o \\
\text { assisted scle } \\
\text { EC were me }\end{array}$ & $\begin{array}{l}\text { ressure; GM=glauc } \\
\text { =right eye; OS=left } \\
\text { s stromal hypoplas } \\
\text { asty; DSAEK=Desc } \\
\text { erior chamber intra } \\
\text { ectomy surgery; NR } \\
\text { sured with ICVM. }\end{array}$ & $\begin{array}{l}\text { ma medi } \\
\text { eye; CCT } \\
\text { a; AS=ant } \\
\text { met's stri } \\
\text { cular len } \\
\text { =needle re }\end{array}$ & $\begin{array}{l}\text { ations; } \\
\text { entral co } \\
\text { ior syne } \\
\text { ping } \\
\text { ision of }\end{array}$ & $\begin{array}{l}\text { neal } \\
\text { niae } \\
\text { ailed }\end{array}$ \\
\hline
\end{tabular}

Additional surgical procedures during keratoplasty included synechiolysis $(n=11)$, phacoemulsification $(n=2)$ and PCIOL implantation $(n=2)$.

\section{Intraoperative and postoperative complications}


All surgical interventions were successful, and no intraoperative complications were noted. There was no immediate markedly elevated IOP, interface fluid or graft detachment. Mean PAS involved 5 clock hours without progressive manifestations.

\section{Graft survival}

Eight of 11 eyes (72.7\%) survived during the follow-up period. One PK graft failed at 21 months, and 2 DSAEK grafts failed at 24 and 45 months (Table 1). One eye received repeat DSAEK (patient 4, left eye) (shown in Fig. 1).

\section{Additional glaucoma treatment after keratoplasty}

In the PK group, 2 of 4 eyes (50.0\%) exhibited high IOP and received additional glaucoma therapy after keratoplasty: 1 eye without glaucoma history was on topical medications alone, while repeat glaucoma surgeries were performed for another eye with glaucoma history (Table 1 ). In the DSAEK group, of these 6 eyes with a history of glaucoma, 5 eyes (83.3\%) required additional glaucoma treatment after keratoplasty: 3 were on topical medications alone, and 2 required glaucoma surgeries. One eye without a history of glaucoma required topical medications after keratoplasty (Table 1).

In general, 4 eyes exhibited markedly high IOP postoperatively and had previous glaucoma surgeries. Eight of 11 eyes (72.7\%) received additional glaucoma treatment after keratoplasty: 2 (50.0\%) in the PK group and 6 (66.7\%) in the DSAEK group (P=0.62). Of these 8 eyes, 1 PK eye (25.0\%) and 2 DSAEK eyes (28.6\%) required glaucoma surgeries. All 3 eyes underwent glaucoma surgeries before keratoplasty.

\section{Visual outcomes}

After keratoplasty, the mean BCVA reached a maximum of $0.7 \pm 0.5$ LogMAR (range, 0.2-2.0 LogMAR) at $8.9 \pm 7.5$ months. The difference between the PK and DSAEK groups in the maximum BCVA and the time approaching the maximum BCVA showed no significant difference (0.7 \pm 0.5 LogMAR vs. $0.7 \pm 0.6$ LogMAR, $P_{1}=1.00 ; 13.5 \pm 9.0$ months vs. $6.3 \pm 5.7$ months, $P_{2}=0.12$ ).

\section{ECD and ECL}

Table 2 and Figure 2 show the outcomes for ECD and ECL rate in the PK group and DSAEK group. The ECD values of donor tissues in the PK and DSAEK groups were not significantly different $(P=0.155)$. Compared with the ECD of donor tissues, the ECL rate during PK and $D S A E K$ was not significant at 1 month $(P=0.18,0.07)$. There was no significant difference between the PK and DSAEK groups in the ECL rate at $1,6,12$ and 24 months $\left(P_{1}=0.64, P_{2}=1.00, P_{3}=0.57\right.$, and $\left.P_{4}=0.44\right)$.

Table 2

Endothelial cell density and endothelial cell loss after keratoplasty

\begin{tabular}{|c|c|c|c|c|c|}
\hline & \multirow[t]{2}{*}{ Baseline } & \multicolumn{4}{|c|}{ Follow-up visit } \\
\hline & & 1 Month & 6 Months & 12 Months & 24 Months \\
\hline ECD (cells $\left./ \mathrm{mm}^{2}\right)$ & $3274 \pm 490$ & $1807 \pm 634$ & $1685 \pm 766$ & $1233 \pm 591$ & $1422 \pm 467$ \\
\hline PK & $2936 \pm 466$ & $1364 \pm 93$ & $1718 \pm 1086$ & $1152 \pm 576$ & $1575 \pm 404$ \\
\hline DSAEK & $3467 \pm 414$ & $2028 \pm 686$ & $1666 \pm 622$ & $1279 \pm 705$ & $1193 \pm 1026$ \\
\hline ECL rate $(\%)$ & - & $43 \pm 15$ & $49 \pm 23$ & $63 \pm 16$ & $54 \pm 20$ \\
\hline PK & - & $47 \pm 13$ & $42 \pm 35$ & $60 \pm 19$ & $45 \pm 14$ \\
\hline DSAEK & - & $41 \pm 17$ & $52 \pm 17$ & $64 \pm 16$ & $67 \pm 25$ \\
\hline
\end{tabular}

The six-month ECL rate and 12-month ECL rate, which were detectable for all 11 eyes, were not significantly correlated with previous glaucoma surgeries $\left(P_{1}=0.73, P_{2}=0.46\right)$, baseline IOP $\left(P_{1}=0.71, P_{2}=0.19\right)$, ACD before keratoplasty $\left(P_{1}=0.43, P_{2}=0.67\right)$, type of keratoplasty $\left(P_{1}=1.00\right.$, $\left.P_{2}=0.60\right)$, or additional glaucoma surgeries after keratoplasty $\left(P_{1}=0.99, P_{2}=0.96\right)$.

\section{Discussion}

The prognosis for corneal grafts in ARS is not established due to a lack of published data [11, 12]. In the current study, we report the clinical outcomes of keratoplasty in 11 consecutive eyes with irreversible corneal decompensation secondary to ARS after a mean 3-year follow-up duration. 
Previously, patients with ARS were considered unsuitable for keratoplasty [18], although corneal decompensation secondary to ARS deprived them of the ability to normal life and work. However, there was no other effective treatment to release these patients from persistent pain and poor vision. Moreover, glaucoma might still be the greatest problem throughout their lifetime even without keratoplasty. Therefore, at great pain and vision loss, keratoplasty gave them an opportunity for restoring vision and hope of life.

The clinical outcomes in this series were encouraging. Both PK and DSAEK can successfully treat corneal dysfunction secondary to ARS, with a graft survival rate of $72.7 \%$ (8/11). Lacking published clinical outcomes of keratoplasty for the treatment of ARS, the surgical outcomes in our series were compared with those in iridocorneal endothelial (ICE) syndrome, which shares similar anterior segment manifestations with ARS and has an approximately $50 \%$ risk of developing glaucoma [19]. Overall, previous case series for keratoplasty in eyes with ICE syndrome included a total of 115 PK cases with an average mean follow-up duration of 57.6 months and 78 DSAEK cases with an average mean follow-up of 35.9 months. According to these publications, the average graft survival rate was $67.0 \%$ in PK and $64.1 \%$ in DSAEK [17, 20-27]. The graft survival rate in our study was favorable. Unlike the progression of synechiae after keratoplasty in ICE syndrome [21, 28], PAS showed no progressive manifestations in our series.

In the current study, $72.7 \%$ of eyes (8/11) required additional glaucoma treatment, which was higher than that in ICE syndrome conducted by a multicenter [26]. This multicenter study including 17 PK eyes and 12 DSAEK eyes with ICE syndrome reported that $37.9 \%$ of eyes received glaucoma therapy after keratoplasty. The higher baseline IOP $(21.6 \pm 8.1 \mathrm{mmHg}$ in our series vs. $14.7 \pm 3.9 \mathrm{mmHg}$ in the multicenter), higher morbidity of glaucoma (63.6\% in our series vs. $55.2 \%$ in the multicenter), and biometrically shallow anterior chamber in Asian eyes might be responsible for the higher rate of IOP elevation after keratoplasty in our series. In our study, the IOP in eyes with previous glaucoma surgeries was more difficult to control after keratoplasty. Of the 8 eyes that required additional glaucoma therapy, 3 eyes showed mildly elevated IOP postoperatively, which was considered to be associated with the long-term use of topical corticosteroids; however, all 4 eyes with dramatically high IOP postoperatively had previous glaucoma surgeries.

The maximum BCVA in our series was similar to that reported in our previous study with ICE syndrome (0.57 LogMAR) but poorer than those in other previous studies ( 0.4 to 0.0 LogMAR) $[24,25,28]$. The difference in visual outcomes among these studies might be attributed to amblyopia and a higher morbidity of glaucoma in the current series and our previous study (63.6\% and 65.0\%) [17]. In our study, DSAEK eyes did not show better BCVA than PK eyes due to the higher morbidity of glaucoma. Although the DSAEK group reached a maximum BCVA more quickly than the PK group (6.3 months vs. 13.5 months), the difference did not reach statistical significance due to the small sample size. Four of 7 (57.1\%) DSAEK eyes underwent keratoplasty in adulthood when irreversible amblyopia had formed. In contrast, BCVA improved over time during the follow-up period in the PK group because all PK patients were children who accepted standard amblyopia treatment after keratoplasty, which contributed to their BCVA sequential improvement. Thus, this result might not be sufficient to indicate that DSAEK allows more rapid visual recovery than PK.

DSAEK techniques show endothelial cell survival similar to that of PK in eyes with ARS, even though DSAEK is more challenging for graft manipulation and endothelial cell protection owing to iridocorneal synechiae and shallow anterior chambers. The decrease in postoperative ECD was prominent at the 1-month review in both PK and DSAEK, which is consistent with previous studies [17, 29, 30]. The complicated anterior segment in eyes with ARS leads to great difficulty in graft manipulation and increased perioperative inflammation, which is an important reason for the increased perioperative ECL $[25,16,28]$.

The study is limited by its retrospective nature and small sample size, primarily due to the rarity of irreversible corneal decompensation secondary to ARS. Although it is the largest series reported for both PK and DSAEK, the long-term clinical outcomes of keratoplasty for ARS remain to be evaluated.

\section{Conclusions}

In conclusion, all the patients returned to normal life after keratoplasty. With a mean follow-up period of 3 years, grafts survived in $72.7 \%$ of ARS patients after keratoplasty, and $72.7 \%$ of eyes required additional glaucoma treatment. Eyes that underwent glaucoma surgeries before keratoplasty were more likely to exhibit markedly high IOP and require additional glaucoma therapy. Both PK and DSAEK resulted in similar outcomes with regard to IOP control, BCVA and ECL. Under sufficient synechiolysis and postoperative glaucoma management, patients with ARS can achieve transparent corneas, visual acuity improvement and pain remission after keratoplasty. Considering the better structural integrity and superiority of repeat keratoplasty with DSAEK, it may be the preferred procedure for irreversible keratoplasty secondary to ARS.

\section{Declarations}

\section{Author's contributions}

JH conceived and supervised the study. All keratoplasty operations were performed by JH. TY and RP collected the data. TY and GX analyzed the data. TY wrote the manuscript. JH was considered corresponding author. TY was considered first author. All authors read and approved the final 
manuscript.

\section{Funding}

Supported by the National Natural Science Foundation of China under Grant No. 81800801 and No. 81970768. The funding organization had no role in the preparation of data and the manuscript.

\section{Conflict of interest}

The authors have no conflicts of interest to declare that are relevant to the content of this article.

\section{Ethical approval}

This study was approved by the Human Research Ethics Committee of Peking University Third Hospital (approval number: IRB00006761M2018244) and was conducted in accordance with the Declaration of Helsinki.

\section{Consent for publication}

Written informed consent was obtained from each participant or their guardian before the data collection.

\section{References}

1. Seifi M, Walter MA (2018) Axenfeld-Rieger syndrome. Clin Genet 93:1123-1130. https://doi.org/10.1111/cge.13148

2. Shields MB (1983) Axenfeld-Rieger syndrome: a theory of mechanism and distinctions from the iridocorneal endothelial syndrome. Trans Am Ophthalmol Soc 81:736-784

3. Chang TC, Summers CG, Schimmenti LA, Grajewski AL (2012) Axenfeld-Rieger syndrome: new perspectives. Br J Ophthalmol 96:318-322. https://doi.org/10.1136/bjophthalmol-2011-300801

4. Alward WL (2000) Axenfeld-Rieger syndrome in the age of molecular genetics. Am J Ophthalmol 130:107-115. https://doi.org/10.1016/s0002-9394(00)00525-0

5. Ito YA, Walter MA (2014) Genomics and anterior segment dysgenesis: a review. Clin Exp Ophthalmol 42:13-24. https://doi.org/10.1111/ceo.12152

6. Lee WB, Jacobs DS, Musch DC, Kaufman SC, Reinhart WJ, Shtein RM (2009) Descemet's stripping endothelial keratoplasty: safety and outcomes: a report by the American Academy of Ophthalmology. Ophthalmology 116:1818-1830. https://doi.org/10.1016/j.ophtha.2009.06.021

7. Price MO, Price FW Jr (2008) Endothelial cell loss after descemet stripping with endothelial keratoplasty influencing factors and 2-year trend. Ophthalmology 115:857-865. https://doi.org/10.1016/j.ophtha.2007.06.033

8. Jordan CS, Price MO, Trespalacios R, Price FW Jr (2009) Graft rejection episodes after Descemet stripping with endothelial keratoplasty: part one: clinical signs and symptoms. Br J Ophthalmol 93:387-390. https://doi.org/10.1136/bjo.2008.140020

9. Banitt MR, Chopra V (2010) Descemet's stripping with automated endothelial keratoplasty and glaucoma. Curr Opin Ophthalmol 21:144149. https://doi.org/10.1097/ICU.0b013e3283360b95

10. Letko E, Price DA, Lindoso EMS, Price MO, Price FW Jr (2011) Secondary graft failure and repeat endothelial keratoplasty after Descemet's stripping automated endothelial keratoplasty. Ophthalmology 118:310-314. https://doi.org/10.1016/j.ophtha.2010.06.032

11. Ketki S, Alex VL (2012) Resolution of bilateral ptosis after reduction of unilaterally elevated intraocular pressure in a child with AxenfeldReiger spectrum disorder. J AAPOS 16:307-308. https://doi.org/10.1016/j.jaapos.2012.02.012

12. Omneya A, Alejandra ER, Jorge LADB, Jorge LADB, Jorge LA (2019) Treatment of chronic and extreme ocular hypotension following glaucoma surgery with intraocular platelet-rich plasma: A case report. Eur J Ophthalmol 29:NP9-NP12. https://doi.org/10.1177/1120672118803515

13. Sridhar J, Yonekawa Y, Kuriyan AE, Joseph A, Thomas BJ, Liang MC et al (2017) Microbiologic spectrum and visual outcomes of acute-onset endophthalmitis undergoing therapeutic pars plana vitrectomy. Retina 37:1246-1251. https://doi.org/10.1097/IAE.0000000000001358

14. Hong Y, Peng RM, Wang M, Qu HQ, Hong J (2013) Suture pull-through insertion techniques for Descemet stripping automated endothelial keratoplasty in Chinese phakic eyes: outcomes and complications. PLoS ONE 8:e61929. https://doi.org/10.1371/journal.pone.0061929 
15. Yang F, Hong J, Xiao G, Feng Y, Peng R, Wang M et al (2020) Descemet Stripping Endothelial Keratoplasty in Pediatric Patients with Congenital Hereditary Endothelial Dystrophy. Am J Ophthalmol 209:132-140. https://doi.org/10.1016/j.ajo.2019.08.010

16. Wu El, Ritterband DC, Yu G, Shields RA, Seedor JA (2012) Graft rejection following descemet stripping automated endothelial keratoplasty: features, risk factors, and outcomes. Am J Ophthalmol 153:949-957e1. https://doi.org/10.1016/j.ajo.2011.10.004

17. Ao M, Feng Y, Xiao G, Xu Y, Hong J (2017) Clinical outcome of Descemet stripping automated endothelial keratoplasty in 18 cases with iridocorneal endothelial syndrome. Eye(Lond) 32:679-686. https://doi.org/10.1038/eye.2017.282

18. Nischal KK (2015) Genetics of Congenital Corneal Opacification-Impact on Diagnosis and Treatment. Cornea 34 Suppl 10 S24-S34. https://doi.org/10.1097/ICO.0000000000000552

19. Laganowski HC, Kerr Muir MG, Hitchings RA (1992) Glaucoma and the iridocorneal endothelial syndrome. Arch Ophthalmol 110:346-350. https://doi.org/10.1001/archopht.1992.01080150044025

20. Buxton JN, Lash RS (1984) Results of Penetrating Keratoplasty in the Iridocorneal Endothelial Syndrome. Am J Ophthalmol 98:297-301. https://doi.org/10.1016/0002-9394(84)90319-2

21. Crawford GJ, Stulting RD, Cavanagh HD, Waring GO 3rd (1989) Penetrating keratoplasty in the management of iridocorneal endothelial syndrome. Cornea 8:34-40

22. Chang PC, Soong HK, Couto MF, Meyer RF, Sugar A (1993) Prognosis for penetrating keratoplasty in iridocorneal endothelial syndrome. Refrac Corneal Surg 9:129-132

23. Alvim PT, Cohen EJ, Rapuano CJ, Chung CW, Pereira ML, Eagle RC Jr et al (2001) Penetrating keratoplasty in iridocorneal endothelial syndrome. Cornea 20:134-140. https://doi.org/10.1097/00003226-200103000-00003

24. Chaurasia S, Ramappa M, Garg P, Garp P, Murthy SI, Senthil S et al (2013) Endothelial keratoplasty in the management of irido-corneal endothelial syndrome. Eye (Lond) 27:564-566. https://doi.org/10.1038/eye.2012.298

25. Fajgenbaum MA, Hollick EJ (2015) Descemet Stripping Endothelial Keratoplasty in Iridocorneal Endothelial Syndrome: Postoperative Complications and Long-Term Outcomes. Cornea 34:1252-1258. https://doi.org/10.1097/IC0.0000000000000530

26. Quek DT, Wong CW, Wong TT, Han SB, Htoon HM, Ho CL et al (2015) Graft Failure and Intraocular Pressure Control After Keratoplasty in Iridocorneal Endothelial Syndrome. Am J Ophthalmol 160:422-429.

.e1

.. https://doi.org/10.1016/j.ajo.2015.05.024

27. Rotenberg M, Downward L, Curnow E, Larkin DF, Tuft SJ (2020) Graft Survival After Penetrating and Endothelial Keratoplasty in Iridocorneal Endothelial Syndrome. Cornea 39:18-22. https://doi.org/10.1097/IC0.0000000000002039

28. Price MO, Price FW Jr (2007) Descemet stripping with endothelial keratoplasty for treatment of iridocorneal endothelial syndrome. Cornea 26:493-497. https://doi.org/10.1097/ICO.0b013e318030d274

29. Price MO, Gorovoy M, Benetz BA, Price FW Jr, Menegay HJ, Debanne SM et al (2010) Descemet's stripping automated endothelial keratoplasty outcomes compared with penetrating keratoplasty from the cornea donor study. Ophthalmology 117:438-444. https://doi.org/10.1016/j.ophtha.2009.07.036

30. Price MO, Gorovoy M, Price FW Jr, Benetz BA, Menegay HJ, Lass JH (2013) Descemet's stripping automated endothelial keratoplasty: threeyear graft and endothelial cell survival compared with penetrating keratoplasty. Ophthalmology 120:246-251. https://doi.org/10.1016/j.ophtha.2012.08.007

\section{Figures}

\section{Figure 1}

Representative photographs of left eye in patient 4. (A) Corectopia, diffuse corneal edema and peripheral opacification presented at the time of corneal decompensation. (B) Central cornea regained clear after DSAEK, with a maximum BCVA of 0.2 LogMAR. (C) Graft failed at 24 months. (D) Nine months after repeat DSAEK, corneal clarity was maintained, with a BCVA of 0.4 LogMAR. 

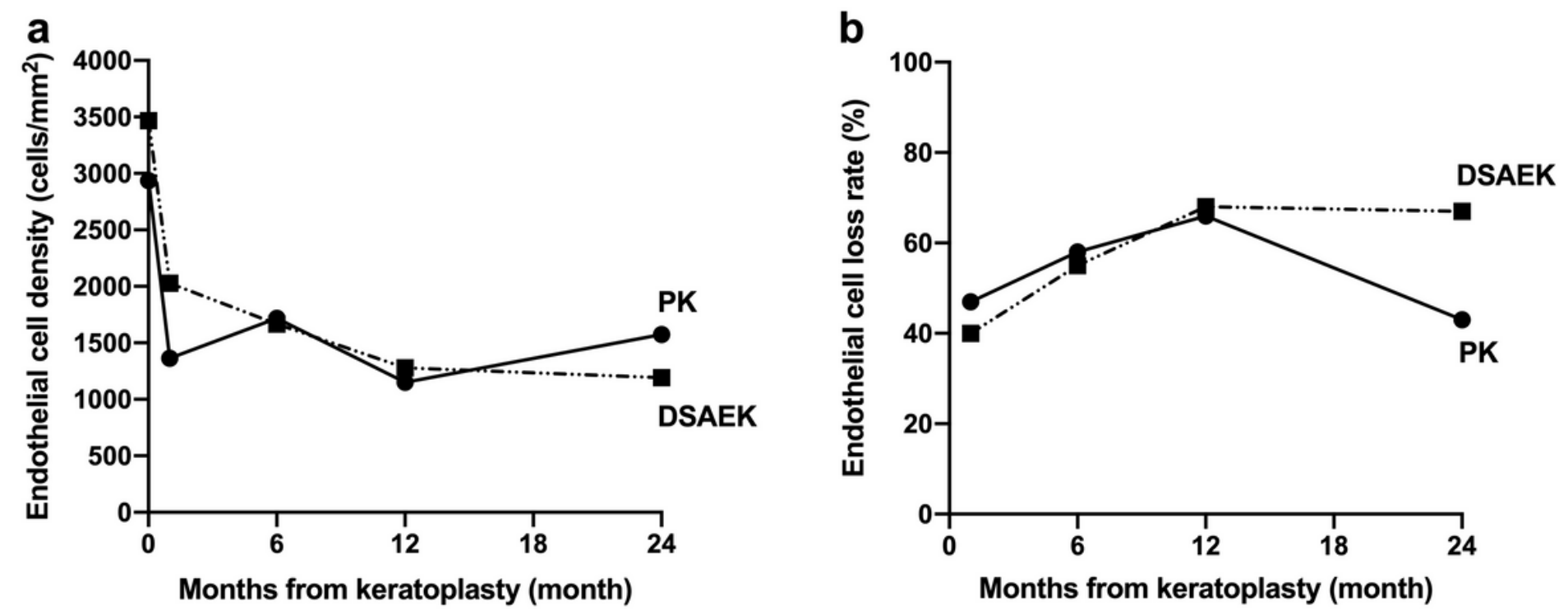

Figure 2

Median endothelial cell density (ECD) and median endothelial cell loss (ECL) rate for surviving grafts over time after keratoplasty. 